\title{
Usefulness of Measuring Thiopurine Metabolites in Children with Inflammatory Bowel Disease and Autoimmunological Hepatitis, Treated with Azathioprine
}

\author{
Katarzyna Bąk-Drabik $\mathbb{D D}^{1},{ }^{1}$ Piotr Adamczyk $\mathbb{D}^{\mathrm{D}},{ }^{2}$ Justyna Duda-Wrońska $\mathbb{D}^{\mathrm{D}}{ }^{3}$ \\ Dominika Dąbrowska-Piechota $\left(\mathbb{D},{ }^{3}\right.$ Anna Jarzumbek $\left(\mathbb{D},{ }^{1}\right.$ and Jarosław Kwiecień $\left(\mathbb{1}{ }^{1}\right.$ \\ ${ }^{1}$ Department of Pediatrics, Faculty of Medical Sciences in Zabrze, Medical University of Silesia, Katowice, Poland \\ ${ }^{2}$ Department of Pediatrics, Faculty of Medical Sciences in Katowice, Medical University of Silesia, Katowice, Poland \\ ${ }^{3}$ Faculty of Medical Sciences in Zabrze, Students Association, Medical University of Silesia, Katowice, Poland \\ Correspondence should be addressed to Katarzyna Bąk-Drabik; bak-drabik@wp.pl
}

Received 7 March 2021; Revised 30 April 2021; Accepted 31 May 2021; Published 18 June 2021

Academic Editor: Muhammad Naeem

Copyright (C) 2021 Katarzyna Bąk-Drabik et al. This is an open access article distributed under the Creative Commons Attribution License, which permits unrestricted use, distribution, and reproduction in any medium, provided the original work is properly cited.

\begin{abstract}
Introduction. Thiopurines, such as azathioprine (AZA) and 6-mercaptopurine (6-MP), are immunomodulatory agents, used for the maintenance of remission in children with inflammatory bowel disease (IBD)_Crohn's disease (CD) and ulcerative colitis (UC), as well as with autoimmunological hepatitis (AIH). Measurements of thiopurine metabolites may allow identifying patients at risk for toxicity and nonadherence. It can also provide an explanation for the ineffectiveness of the treatment, observed in some patients. Patients and Methods. A retrospective analysis was carried out of sixty-eight patients (thirty-six patients with CD, eighteen with UC, and fourteen with AIH), treated with AZA. Thiopurine metabolites, 6-thioguanine nucleotide (6-TGN) and 6methylmercaptopurine (6-MMP), were assayed by high-performance liquid chromatography (HPLC), and the AZA dose was adjusted when 6-TGN concentration was known. Result. Only twenty-five (41\%) children had therapeutic 6-TGN concentrations, ten (16\%) subjects had suboptimal 6-TGN concentrations, and twenty-six subjects (43\%) had 6-TGN concentrations above the recommended therapeutic range. 6-MMP was not above the therapeutic range in any case. Seven subjects revealed undetectable 6-TGN and 6-MMP levels, indicating nonadherence. The mean AZA dose after the 6-TGN concentration-related adjustment did not differ, in comparison to the initial dose, either in IBD or AIH groups. The mean AZA dose was lower in AIH than in IBD. The subjects with an optimal 6-TGN level presented with a higher ratio of remission (88\%) than the under- or overdosed patients $(60 \%$ and $69 \%)$, respectively (Chi-square test $=3.87, p<0.05$ ). Conclusion. Timely measurements of thiopurine metabolites can be a useful tool to identify nonadherent patients before a decision is taken to switch to another drug. We may also spot the patients who receive either too low or too high doses, compensating dose deviations in an appropriate way. The patients with optimal 6-TGN levels presented a higher percentage of remission than the under- or overdosed patients. In most patients, both initial and adjusted AZA doses, lower than suggested in guidelines, appeared to be sufficient to maintain remission.
\end{abstract}

\section{Introduction}

Immunosuppressants are crucial drugs for the treatment of autoimmune disorders, including autoimmune hepatitis (AIH) and inflammatory bowel diseases (IBD). Azathioprine
(AZA) has been proven to be a suitable medication with regard to its efficacy and side-effect profile.

AZA was synthesized in 1957 as a derivative of 6mercaptopurine (6-MP) but earlier, in 1951, George Herbert Hitchings and Gertrude Elion discovered 6-MP and 
thioguanine (TG) as a result of searching for antimetabolites of nucleic acid bases that could arrest cell proliferation [1]. Thiopurines are prodrugs, metabolised by, at least, four different pathways until the final molecules, called thioguanine nucleotides (TGN), are obtained [1].

The metabolism of 6-MP involves three competing pathways: the first one being a degradation to thiouric acid (TUA), which is then excreted, the second one leads through methylation by thiopurine S-methyltransferase (TPMT) into 6-methylmercaptopurine (6-MMP), and the third one involves the breakdown of 6-MP into thioinosine monophosphate (TIMP), catalysed by hypoxanthine phosphoribosyltransferase (HPRT). TIMP is then further metabolised via inosine monophosphate dehydrogenase (IMPDH) into thioguanine triphosphate (TGMP). Kinases convert this into the TGNs [2]. TGNs are the active metabolites which exert immunomodulatory effects, whereas 6-MMP and 6MMPR are the inactive and potentially toxic metabolites. These processes are presented in Figure 1.

AZA and 6-MP are immunosuppressants with short half-lives ( 3 and 1.5 hours, respectively) and, therefore, measuring their metabolites is a more appropriate method, both for adherence assessment and therapeutic drug monitoring. An intracellular accumulation of AZA/6-MP metabolites occurs over a period of 2-3 weeks [3]. Various studies have examined the relationship between 6-TGN levels in red blood cells and a clinical response to thiopurine therapy. There is an evidence that 6-TGN levels above 230 $\mathrm{pmol} / 8 \times 10^{8}$ erythrocytes correspond to a good clinical effect $[4,5]$, however, they do not guarantee remission. On the other hand, a 6 -TGN level above $450 \mathrm{pmol} / 8 \times 10^{8}$ erythrocytes may lead to an increased risk of myelotoxicity [6]. The concentrations of 6 -MMP above $5400 \mathrm{pmol} / 8 \times 10^{8}$ erythrocytes have been related to the development of hepatotoxicity [7]. Thiopurine metabolite measurements become more and more available, although their routine use is still limited by costs and technical requirements at laboratories. The primary aim of this study was to assess the usefulness of monitoring thiopurine metabolites in paediatric patients with $\mathrm{IBD}$ and $\mathrm{AIH}$ to assess their adherence to therapy and treatment safety. According to our knowledge, evaluating the use of metabolite measurement in children receiving thiopurine treatment was not performed in Polish children population. It is probably that the population from which the sample comes from could influence the results of the study. The secondary aim of the study was to compare AZA doses in both diseases and among the subgroups of patients, stratified according to the disease activity.

\section{Material and Methods}

2.1. Subjects. Sixty-eight children (thirty-one girls) with IBD and $\mathrm{AIH}$, receiving azathioprine therapy in a consistent dose to maintain remission for at least 3 months, treated in one regional paediatric gastroenterology centre between April 2017 and May 2020, were identified by means of a retrospective review of their medical records. Within that group, there were thirty-six patients with CD, eighteen with UC, and fourteen with AIH. All the IBD children were treated according

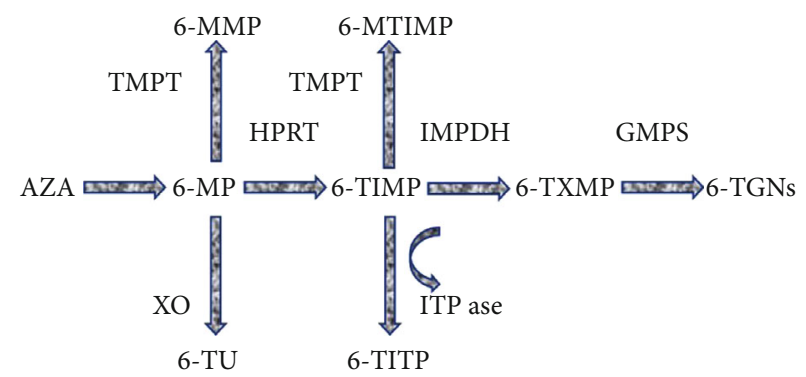

FIgURe 1: Azathioprine metabolism. AZA: azathioprine; HPRT: hypoxanthine phosphoribosyltransferase; IMPDH: inosine monophosphate dehydrogenase; GMPS: guanosine monophosphate synthase; ITPase: inosine triphosphatase; XO: xanthine oxidase; TPMT: thiopurine S-methyltransferase; 6-MP: 6-mercaptopurine; 6-MMP: 6-methylmercaptopurine; 6-MTIMP: 6-methylthioinosine monophosphate; 6-TXMP: 6-thioxanthylic acid; 6-TGNs: 6 thioguanine nucleotides; 6-TIMP: 6-thioinosine monophosphate; 6TITP: 6-thioinosine triphosphate; 6-TU: 6-thiouric acid.

to the ECCO (European Crohn's and Colitis Organization) guidelines $[8,9]$, and the AIH children were treated according to ESPGHAN Hepatology Committee [10]. Seven patients with undetectable 6-TGN and 6-MMP levels were excluded from a detailed analysis concerning the assessment of the mean values of the AZA dose, initial and after correction, 6-TGN, 6-MMP, and a statistical analysis of those variables.

Among the remaining subjects, the following data were collected: demographics, body mass, type of disease, and laboratory data including white blood cell count, haemoglobin, aspartate aminotransferase (AST), alanine transaminase (ALT), and amylase and thiopurine metabolites (6-TGN, 6MMP). IBD activity was determined, using the respective scales: PUCAI (the Paediatric Ulcerative Colitis Activity Index) and PCDAI (the Paediatric Crohn's Disease Activity Index). Biochemical remission in $\mathrm{AIH}$ was defined as a normalisation of transaminase activity and IgG concentration [10]. The mean duration of AZA therapy, before azathioprine metabolites were assayed, was 397 days (the range: 127-1294). The characteristics of the study group are presented in Table 1.

2.2. Methods. Azathioprine metabolite (6-TGN and 6-MMP) levels were determined at an external analytical laboratory. In summary, cells, isolated from venous EDTA blood samples, were first three times washed with an isotonic buffer and then lysed, using the thermal disruption method. Subsequently, the lysates were deproteinised by incubation in acidic conditions and centrifuged for at least $15 \mathrm{~min}$. at >10 $000 \mathrm{rcf}$ to remove cellular debris. The cleared lysates were analysed by high-performance liquid chromatography (HPLC) against a reversed-phase (RP) and by detection at 300-350 nm (using a UV-VIS detector). The obtained concentrations were quantified, using the AUC (area under curve) method, comparing the values against a standard curve, obtained with synthetic calibrators of known concentrations. Such raw reads were normalised, based on the RBC (red blood cell count) of each sample. The final results were calculated as $\mathrm{pmol} / 8 \times 10^{8}$ 
TABLE 1: Background information about the group receiving azathioprine.

\begin{tabular}{|c|c|}
\hline Characteristics & Values \\
\hline Total number of patients & 61 \\
\hline Females (percent) & $27(44 \%)$ \\
\hline Age (years \pm SD) & $14.97 \pm 2.6$ \\
\hline Crohn's disease (CD) & $31(50 \%)$ \\
\hline Ulcerative colitis (UC) & $16(26 \%)$ \\
\hline Autoimmune hepatitis (AIH) & $14(23 \%)$ \\
\hline Weight SDS (mean \pm SD) & $0.13 \pm 1.11$ \\
\hline $\mathrm{CD}$ & $-0.23 \pm 1.0$ \\
\hline $\mathrm{UC}$ & $-0.39 \pm 0.7$ \\
\hline $\mathrm{AIH}$ & $0.53 \pm 0.8$ \\
\hline Height SDS $($ mean \pm SD) & $-0.43 \pm 0.8$ \\
\hline $\mathrm{CD}$ & $-0.54 \pm 0.9$ \\
\hline UC & $-0.60 \pm 0.7$ \\
\hline $\mathrm{AIH}$ & $0.11 \pm 0.7$ \\
\hline BMI SDS (mean \pm SD) & $0.06 \pm 0.9$ \\
\hline $\mathrm{CD}$ & $-0.04 \pm 0.9$ \\
\hline UC & $-0.12 \pm 0.8$ \\
\hline $\mathrm{AIH}$ & $-0.03 \pm 2.7$ \\
\hline $\begin{array}{l}\text { Premonitoring azathioprine dose } \\
\mathrm{mg} / \mathrm{kg} \text { (mean } \pm \mathrm{SD})\end{array}$ & $1.15 \pm 0.3$ \\
\hline $\mathrm{CD}$ & $1.22 \pm 0.3$ \\
\hline UC & $1.23 \pm 0.3$ \\
\hline $\mathrm{AIH}$ & $0.94 \pm 0.3$ \\
\hline $\begin{array}{l}\text { Postmonitoring azathioprine dose } \\
\mathrm{mg} / \mathrm{kg}(\text { mean } \pm \mathrm{SD})\end{array}$ & $1.08 \pm 0.4$ \\
\hline $\mathrm{CD}$ & $1.11 \pm 0.4$ \\
\hline UC & $1.26 . \pm 0.3$ \\
\hline AIH & $0.84 \pm 0.3$ \\
\hline 6-TGN $($ mean $\pm S D)$ & $494.7 \pm 345.3$ \\
\hline $\mathrm{CD}$ & $534.9 \pm 371.8$ \\
\hline $\mathrm{UC}$ & $371.0 \pm 174.5$ \\
\hline $\mathrm{AIH}$ & $535.6 \pm 398.4$ \\
\hline 6-MMP $($ mean \pm SD) & $1288 \pm 886.0$ \\
\hline $\mathrm{CD}$ & $1236 \pm 816$ \\
\hline $\mathrm{UC}$ & $1505 \pm 990$ \\
\hline $\mathrm{AIH}$ & $1175 \pm 952$ \\
\hline \multicolumn{2}{|l|}{ Disease activity: } \\
\hline Remission/mild form of IBD & $32(68 \%)$ \\
\hline Moderate form of IBD & $10(21 \%)$ \\
\hline Severe form of IBD & $5(11 \%)$ \\
\hline Remission of AIH & $14(100 \%)$ \\
\hline
\end{tabular}

SD: standard deviation; 6-MMP: 6-methylmercaptopurine; 6-TGN: 6-thioguanine; IBD: inflammatory bowel disease; AIH: autoimmunological hepatitis; CD: Crohn's disease; UC: ulcerative colitis. erythrocytes. Figure 2 presents a typical spectrum, obtained for azathioprine metabolite measurement using HPLC resolved using water to methanol biphasic system.

2.3. Interpretation of the Results. The thresholds of 6-TGN and 6-MMP measurements and the interpretation of obtained results are presented in Table 2. Thus, it was aimed at keeping 6 -TGN levels in the range of $230-450 \mathrm{pmol} / 8 \times 10^{8}$ erythrocytes and 6-MMP below $5700 \mathrm{pmol} / 8 \times 10^{8}$ erythrocytes.

\section{Statistical Analysis}

A statistical analysis was performed, using the Statistica software (StatSoft, Tulsa, OK, USA). Descriptive statistics for continuous variables were presented as mean values and standard deviations. The Shapiro-Wilk test was applied to verify the normality of data distribution. For a comparative analysis (comparisons between study groups), the applied statistical tools included the Student's $t$-test for independent samples or the Mann-Whitney $U$-test (for data with normal or abnormal distribution). The analysis of variance (ANOVA) with a post hoc least significance difference (LSD) test was used when more than 2 subgroups were compared. The longitudinal comparisons between the measurements, obtained at baseline and during follow-up, were assessed by the $t$-test for dependent samples or the Wilcoxon signed-rank test, whichever was appropriate, according to the data distribution. A correlation analysis was done by Pearson's or Spearman's correlation tests, whichever was appropriate, according to data distribution. Qualitative features were presented, juxtaposing the number of subjects with the percentage values in the defined subgroups. Comparisons of qualitative feature prevalence rates were performed by the Chi-square test. Significance for results in all the statistical analyses was assumed at $p<0.05$.

The caregivers of the patients have consented to the use of their medical data in anonymised forms for statistical, educational, and scientific purposes, which is a standard procedure at the hospital. The current data analysis has been approved by the hospital authorities.

\section{Results}

4.1. Measurements of 6-TG and 6-MMP: An Interpretation of Metabolite Levels in the Group Receiving AZA. The mean 6TGN values in the whole group, as well as in CD, UC, and AIH patients, were $494.7 \pm 343.5,534.9- \pm 371,8371.0 \pm$ 174.5 , and $535.6 \pm 398.4 \mathrm{pmol} / 8 \times 10^{8}$ erythrocytes, respectively. The mean 6-MMP values in the whole group, as well as in CD, UC, and AIH patients, were $1288 \pm 886,1236 \pm$ $816,1505 \pm 990$, and $1175 \pm 952 \mathrm{pmol} / 8 \times 10^{8}$ erythrocytes, respectively (see Figure 3 ).

Twenty-five (41\%) children had therapeutic 6-TGN concentrations with a normal 6-MMP range. Ten (16\%) had suboptimal 6-TGN concentrations with a normal 6-MMP range, which indicates that the AZA dose was below the therapeutic level. Twenty-six subjects (38\%) had 6-TGN concentrations above the required therapeutic range with a normal 6-MMP concentration, which indicated hypomethylation 

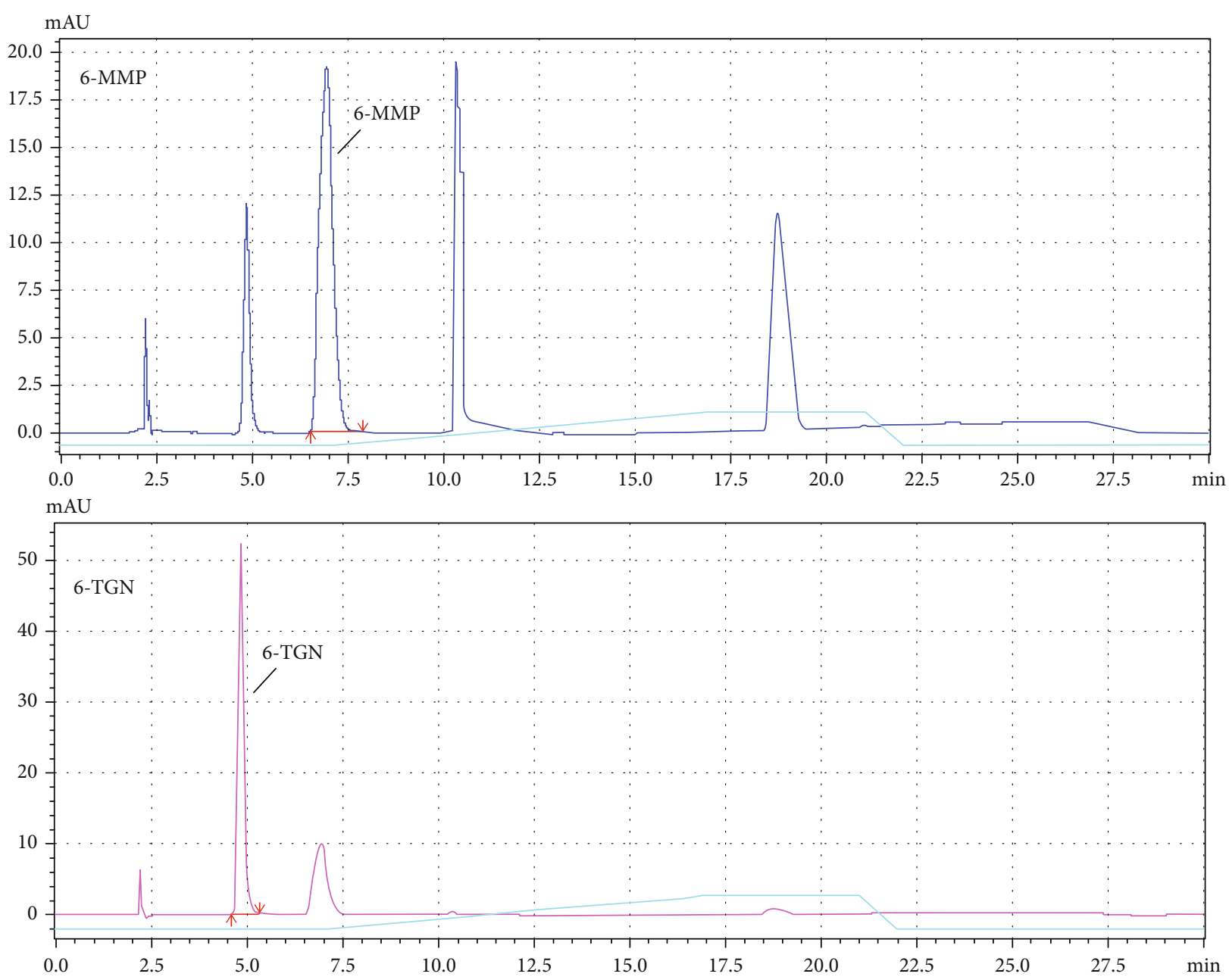

FIGURE 2: Representative examples of full HPLC spectra acquired for the measurements of thiopurine metabolites, 6-MMP (top) and 6-TGN (bottom). The marked peaks are specific for 6-MMP and 6-TGN, respectively. The remaining peaks do not have an influence on result interpretation.

TABLE 2: Interpretation of metabolite levels (measured in pmol/8 $\times 10^{8}$ erythrocytes) and recommended approaches. TPMT: thiopurine methyltransferase; 6-MMP: 6-methylmercaptopurine; 6-TGN: 6-thioguanine.

\begin{tabular}{|c|c|c|c|}
\hline 6-TGN & 6-MMP & Interpretation & Recommendation \\
\hline Very low & Very low & Nonadherence & Improve adherence \\
\hline Low $(<230)$ & Normal $(<5700)$ & Insufficient dose & Consider dose increase \\
\hline Normal (230-450) & Normal $(<5700)$ & Therapeutic optimum & Further monitoring of treatment \\
\hline High $(>450)$ & High $(>5700)$ & Overdosing & Dose reduction \\
\hline Low $(<230)$ & High $(>5700)$ & $\begin{array}{l}\text { Hypermethylation, risk of } \\
\text { hepatotoxicity }\end{array}$ & $\begin{array}{l}\text { Consider changing treatment or adding } \\
\text { allopurinol with low doses of AZA }\end{array}$ \\
\hline High $(>450)$ & Normal $(<5700)$ & $\begin{array}{l}\text { Potential TPMT deficiency, risk } \\
\text { of myelotoxicity }\end{array}$ & $\begin{array}{l}\text { Monitoring blood test, consider } \\
\text { dose reduction }\end{array}$ \\
\hline Normal (230-450) & High $(>5700)$ & $\begin{array}{l}\text { Hypomethylation, risk of } \\
\text { hepatotoxicity }\end{array}$ & $\begin{array}{c}\text { Monitoring liver enzymes, split or reduce } \\
\text { the dose }\end{array}$ \\
\hline$>1000$ & Undetectable & $\begin{array}{l}\text { Potential TMPT absence, lack of } \\
\text { methylation, risk of acute toxicity }\end{array}$ & Discontinuation of treatment \\
\hline
\end{tabular}




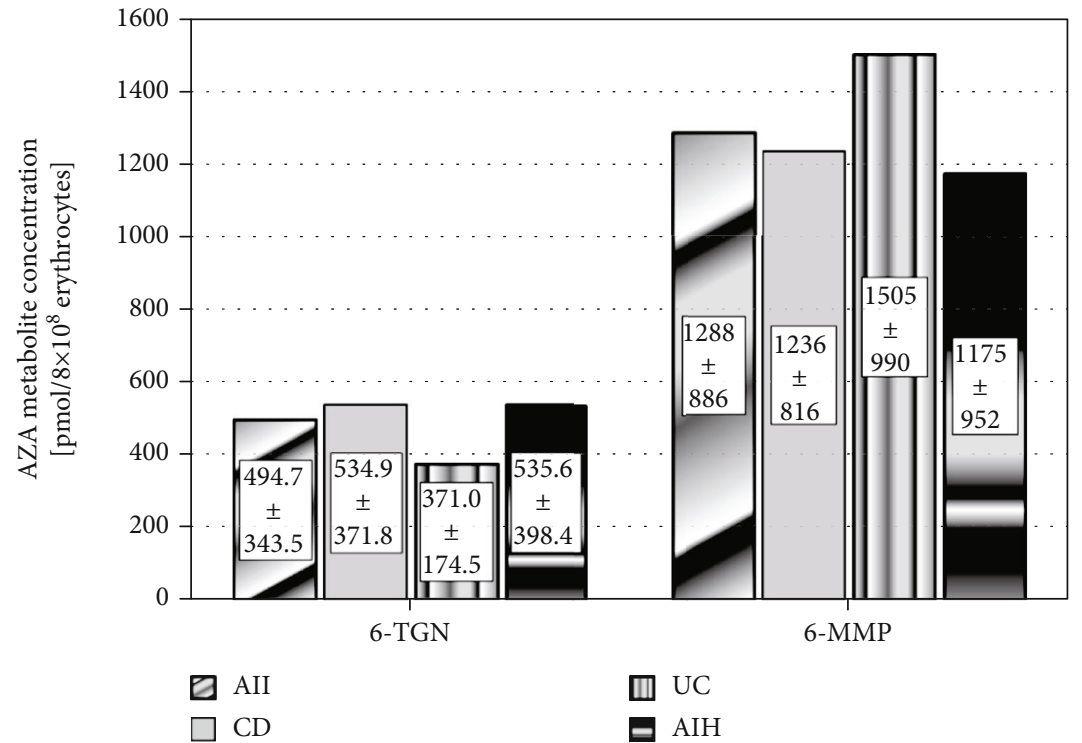

FIGURE 3: Interpretation of metabolite levels in the group receiving AZA. AZA: azathioprine; CD: Crohn's disease; UC: ulcerative colitis; AIH: autoimmunological hepatitis; 6-MMP: methylmercaptopurine; 6-TGN: 6-thioguanine.

and a potential toxicity for the bone marrow. Seven subjects had undetectable 6-TGN and 6-MMP levels, which indicated nonadherence to the therapy. 6-MMP concentrations above the range, which would indicate potential hepatotoxicity, were not identified in any case (see Table 3 ).

\subsection{The Mean AZA Dose, Initial and after Adjustment}

\subsubsection{The Difference between Pre- and Postadjustment of} $A Z A$ Dose, Both in IBD (CD,CU) and AIH Subjects. The presented mean initial AZA dose in the whole study group, as well as in $\mathrm{CD}, \mathrm{UC}$, and AIH subgroups, was $1.15 \pm 0.35$, $1.22 \pm 0.37, \quad 1.23 \pm 0.32$, and $0.94 \pm 0.30 \quad(\mathrm{mg} / \mathrm{kg} /$ day $)$, respectively. The dose after adjustment, based on 6-TGN concentrations, did not differ significantly from the initial AZA dose, either in the whole study group or in $\mathrm{CD}, \mathrm{UC}$, or $\mathrm{AIH}$, and was $1.08 \pm 0.44,1.11 \pm 0.4,1.26 \pm 0.32$, and $0.84 \pm 0.31(\mathrm{mg} / \mathrm{kg} /$ day), respectively (see Figure 4). Neither was there any difference between the girls and the boys nor between the subgroups, defined according to the various methods of treatment or the disease activity, neither at baseline nor at the second assay.

4.2.2. The Difference between IBD (CD, UC) and $A I H$ Subjects, Both for Pre- and Postadjustment AZA Doses. A significant difference was revealed between IBD (CD, UC) and AIH subjects for the pre- and postadjustment AZA doses $(p<0.05)$, namely, the mean AZA dose was lower in AIH than in IBD patients. There was no correlation between the initial AZA dose and 6-TGN levels; after dose adjustment, based on 6-TGN concentrations that correlation could be clearly observed $(R=-0.43, p<0.005)$.

4.3. The Effect of Metabolite Measurements on Dose Modification in the Group Enrolled to the Study $(n=68)$. In 7 cases, the level was undetectable and AZA was reintroduced. In $46 \%(28 / 61)$ cases, the dose was not changed. In the other cases (55\%), the AZA dose was corrected. In $16 \%$ (10/61) cases, the AZA levels were below the range but the dose was increased only in 15\% (9/61) subjects because of slightly decreased levels of leucocytes in the remaining patients of the study group. In $42 \%$ cases (26/61), 6-TGNlevels were above the range but dose modification was introduced only in $39 \%(24 / 61)$ subjects. In two patients, 6-TGN concentration was slightly above the range; the dose was maintained for the lack of remission (see Figure 5).

The subject with optimal 6-TGN levels presented a higher ratio of remission (88\%) than those who were either under- or overdosed $(60 \%$ and $69 \%)$, respectively (Chi - square test $=3.87, p<0.05)$.

4.4. Adverse Outcomes. One patient (1.6\%) developed leucopoenia $\left(<3.5 \mathrm{WBCx} \times 10^{9}\right)$, while none of the studied subjects developed any elevation of the liver enzymes. No other side effects, such as pancreatitis, glomerulonephritis, or lymphoma, were found in any of the patients throughout the study period.

4.5. A Correlation between 6-TGN Levels and Faecal Calprotectin. No correlation was found between faecal calprotectin and 6-TGN levels.

\section{Discussion}

Monitoring of thiopurine metabolites is a part of the safe treatment strategy and, together with other actions, such as the pretreatment screening for virus infections, a routine monitoring of leucocytes and aminotransferase, dose splitting strategies, allopurinol supplementation, and testing for TPMT deficiency, it helps reduce the risk of side-effects [11].

5.1. Nonadherence Rate. 6-TGN levels are useful to identify nonadherence to thiopurine therapy, and it has been suggested that routine observations of the metabolites can help 
TABle 3: Metabolite levels. Mean standard deviations and the range of values of 6-thioguanine (6-TGN), 6-methylmercaptopurine (6-MMPN), measured in $\mathrm{pmol} / 8 \times 10^{8}$ erythrocytes.

\begin{tabular}{lcc}
\hline 6-TGN levels (all subjects) & 6-MMPN levels (all subjects) & Interpretation \\
\hline Mean level: $494 \pm 343$ & $1288 \pm 886$ & Therapeutic optimum \\
Within range $41 \%(25 / 61)$ & Within range & Insufficient dose \\
Below range $16 \%(10 / 61)$ & Within range & Potential TPMT deficiency \\
Above range $43 \%(26 / 61)$ & Within range & (potential bone marrow toxicity) \\
Undetectable 7 subjects & Undetectable & Nonadherence \\
\hline
\end{tabular}

6-MMP: methylmercaptopurine; 6-TGN: 6-thioguanine; TPMT: thiopurine S-methyltransferase.

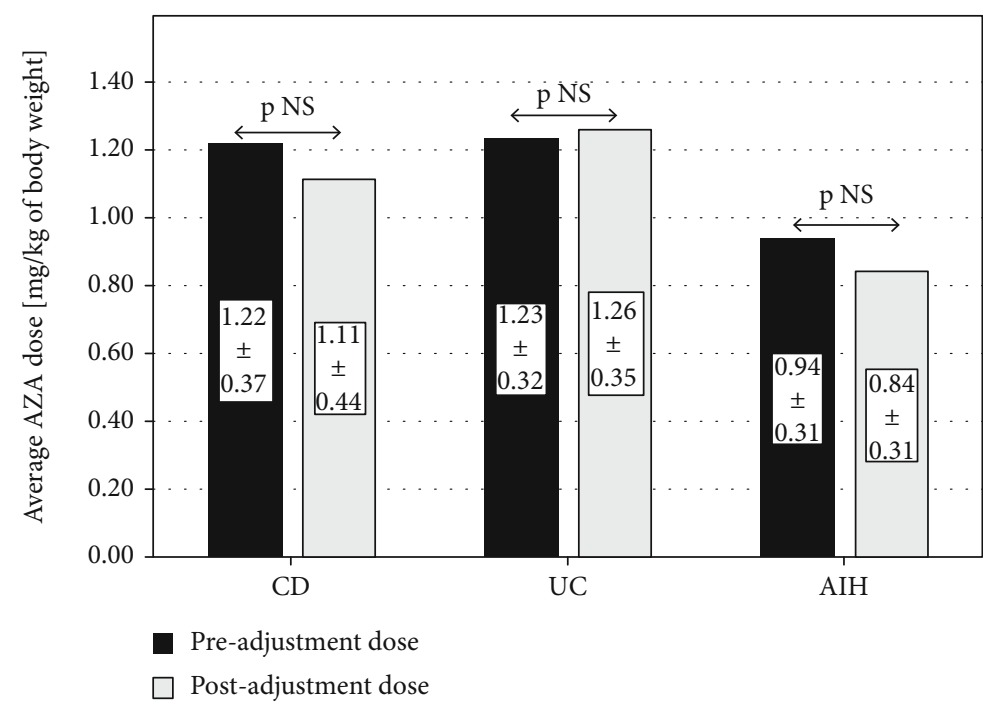

FIgUre 4: The difference between pre- and postadjustment AZA doses, both in IBD (CD, UC) and AIH subjects. AZA: azathioprine; CD: Crohn's disease; UC: ulcerative colitis; AIH: autoimmunological hepatitis.

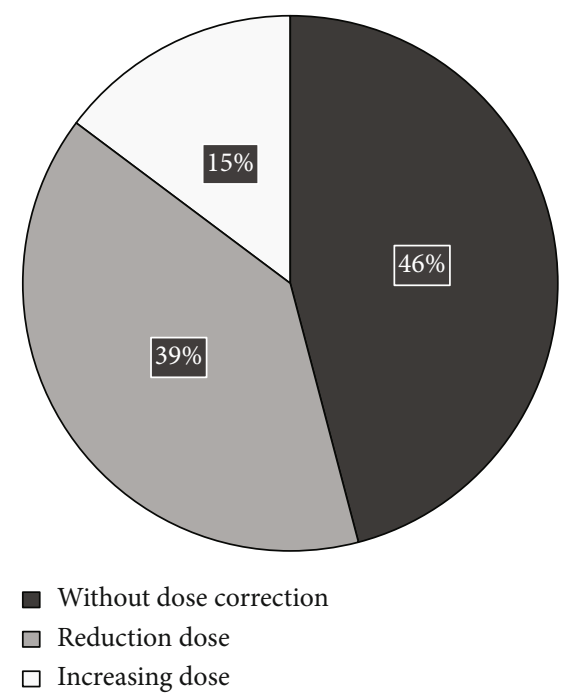

FIGURE 5: Therapeutic decision based on 6-TG concentrations. 6TGN: 6-thioguanine. improve the adherence rates [12]. Several factors, such as sociodemographic, individual, family, disease regimen, and health care system, influence nonadherence. Indeed, in our study, only in $46 \%$ of cases, 6-TGN levels were within the therapeutic range and the dose was not changed. In that group of patients, the subjects with optimal 6-TGN levels presented higher a higher remission percent (88\%) than those who were either under- or overdosed. That observation applied to all the $\mathrm{AIH}$ and IBD subjects. In a large study, where metabolite levels were reviewed in 9187 patients, the therapeutic goal was achieved only in 2444 patients $(27 \%)$ [13].

In our study, seven subjects had undetectable 6-TGN and 6-MMP levels. A detailed medical history, regarding medicine intake regularity, revealed that those patients had not been taking the prescribed medicines for fear of side effects. In that group, there were five $\mathrm{CD}$ and two UC patients. We did not asses the adherence to treatment, using any specific questionnaires, although a multimethod assessment is more widely used [3]. In the future, we plan to assess adherence not only by objective methods but also with a specific questionnaire for both: parents and children. The lack of remission was an indication to consider the reintroduction of 
AZA therapy. In a Spanish study, the authors did not find any high rates of nonadherence $(6.45 \%)$ but they strongly emphasised that the measurements of thiopurine metabolite concentrations could be useful to identify nonresponders before replacing or combining thiopurines with other alternative treatments (generally biological agents), with a consequent increase in both, a potential toxicity and costs [2]. Bokemeyer et al. [14] revealed in their study that, in a group of 65 adult CD patients, six (9.2\%) had metabolite profiles that were indicative of nonadherence. The rate of nonadherence is comparable to the values in previously published studies $[13,15]$. Hommel et al., evaluating adherence in 42 IBD adolescents, found that the majority of the sample (93\%) had demonstrated quantifiable 6-TGN levels but only $14 \%$ were within the therapeutic range what indicated that nonadherence assessment was especially important in the group of adolescents, faced with learning to manage a chronic condition and negotiate normal developmental issues [16]. Alsous et al. [3], using a binary logistic regression analysis, identified the age to be independently predictive of adherence, with adolescents more likely to be classified as nonadherent. The mean age of our nonadherent subjects was 15 years. The patients, who are nonadherent, are more likely to have a more severe course of disease' potentially necessitating the need for a more aggressive medical treatment, such as an increased corticosteroid use or surgery, present a higher risk of disease recurrence, in addition to these medical consequences, and, eventually, suffer of poor psychosocial functioning and low quality of life [17].

5.2. Underdosing. The regular measurements of metabolites can also identify patients who receive too low or too high drug dose, with available information about thiopurine methyltransferase (TPMT). In the Caucasian population, $0.3 \%$ subjects have TMPT deficiency, $6-11 \%$ have moderately reduced levels of TPMT activity, and $89-94 \%$ have normal TMPT activity. Tests for TPMT deficiency, prior to the onset of thiopurine therapy, should be the first step in personalising thiopurine therapy; however, cytopenia may still occur, despite normal TPMT activity, which does not identify patients at risk of other toxic or allergic adverse events, either. The latter information may help differentiate patients between those who have received a suboptimal AZA dose and those who have had higher TPMT activity, shifting AZA metabolism towards 6-MMP production. However, the cost and availability significantly reduce the use of the tests in routine practice. For this reason, we did not perform this test before the beginning of treatment at our hospital.

In our study, 10 cases (15\%) had 6-TGN levels below the therapeutic range with 6-MMP within the range that indicated underdosing. It could also indicate irregular medication intake, so a detailed medical history is essential. 6-TG below the therapeutic range and 6-MMP above the range could indicate preferential metabolism via the TPMT pathway but it was not observed in our study. Another study showed even a higher percentage of underdosing [46].

It is recommended to keep 6-TGN levels between 250 and 450 pmol to maintain remission in inflammatory bowel disease $[8,9,18,19]$. In one of the recent studies, it was revealed that serial thiopurine metabolite level assessments and dose adjustment aiming to maintain higher 6-TGN levels could be helpful to improve long-term outcomes in patients with IBD. The median 6-TGN levels were significantly higher in the patients who did not relapse, as compared with the levels in those patients who did relapse (233 vs. $167 \mathrm{pmol}$ per $8 \times 10^{8}$ erythrocytes, $p=0.025$ ) [5]. Dubinsky et al. demonstrated that, in paediatric patients, $6-$ TGN level $\geq 235$ pmol per $8 \times 10^{8}$ erythrocytes was associated with a therapeutic response to 6-MP [20]. Wright et al. also revealed that those patients, who developed active disease, accumulated significantly lower 6-TGN concentrations than those who remained in remission ( 175 vs. 236 pmol per $8 \times 10^{8}$ erythrocytes, respectively). This study shows additionally that, due to intrapatient variability in 6-TGN production and the high incidence of compliance problems, a single 6-TGN reading may not be reflective of drug metabolism and serial measurements could be more useful [15].

All our children, whose 6-TGN level was below the therapeutic range, were IBD patients. They reported a regular intake of AZA, so AZA dose was increased. An ideal therapeutic 6-TGN-level for AIH was not determined [21]. The abovementioned Sheiko study revealed that $87 \%$ of 66 children maintained sustained biochemical remission in association with low 6-TGN levels, ranging from 50 to $250 \mathrm{pmol}$ [21]. In a French study [22], the subjects in remission had similar-6-TGN levels (mean 6-TGN $436 \mathrm{pmol}$ ) as those with active disease (mean 6-TGN 406 pmol), which demonstrated the lack of correlation between 6-TGN levels and remission induction. After dose modification, follow-up measurements were carried out after three months.

5.3. Overdosing. In 39\%, 6-TGN levels were above the range, with 6-MMP levels within the range, which indicated a potential TPMT deficiency and potential bone marrow toxicity. We did not observe $6-$ TGN $>1000$ pmol per $8 \times 10^{8}$ erythrocytes with undetectable 6-MMP, which could suggest TPMT absence. A high concentration of 6-TGN is associated with an increased occurrence of adverse events. In a study by Lee et al. [23], the occurrence of leucocytopaenia and lymphopenia was associated with high concentrations of 6TGN. Also, Pavlovska et al. showed similar results [24]. However, we did not observe this correlation in our study; the possibility of serious side effects should be considered in case of high 6-TGN levels. An interpretation of the range as high ( $>450$ pmol per $8 \times 10^{8}$ erythrocytes) depends on clinical features. In cases of active diseases, high 6-TGN levels suggest a thiopurine refractory case, prompting for an alternative treatment [25]. In case of remission or mild disease, dose reduction should be considered. In our study, dose modification was decided in 24 patients (39\%), and in 2 patients, 6-TGN concentration was only slightly above the range, so the dose was maintained.

As in some other studies, we did not find any correlation between thiopurine dose and 6-TGN levels; therefore, increasing the drug dose may not be sufficient to reach the desired 6 -TGN target $[24,26]$. This may be explained by an increased methylation of intermediate 6-MP metabolites by inherited high levels of TPMT activity [5]. Other 
explanations refer to changes in azathioprine absorption, depending on disease activity, AZA formulation, or interactions with other drugs, such as mesalazine or and sulphasalazine [27]. On the contrary, Lee et al. found a positive correlation between the dose of AZA and the concentrations of 6-TGN $(p<0.0001)$ [23]. Despite the trend, favouring individualised dosing, other studies show no statistically significant differences in treatment efficacy between individualised dosing, based on baseline TPMT activity, and dosing, subsequently adjusted, according to the 6-TGN concentrations and weight-based AZA dosing [28].

5.4. Mean AZA Dose. In our study, the mean initial AZA dose and the dose after adjustment, based on 6-TGN concentrations, were lower than those, proposed in ECCO and ESPGHAN guidelines, both in IBD and AIH patients.

In AIH patients, the initial AZA dose was $0.5 \mathrm{mg} / \mathrm{kg} /$ day and then it was increased up to a maximum of 2.0-2.5 $\mathrm{mg} / \mathrm{kg} /$ day. Our observation was similar to that in another study. Sheiko et al. [21] revealed that AZA dose of approximately $1.2-1.6 \mathrm{mg} / \mathrm{kg} /$ day was sufficient to maintain biochemical remission in the majority of patients. There was no correlation between AZA dose and 6-TGN levels, which was a similar conclusion to that in our study. The AZA dose in AIH was significantly lower, not only than proposed by ESPGHAN but also than that in IBD subjects. Those observations are coherent with Sheiko observation [22].

In inflammatory bowel diseases (CD and UC), the recommended dose is $2.0-2.5 \mathrm{mg} / \mathrm{kg}$, and for its prodrug, 6mercaptopurine, $1.0-1.5 \mathrm{mg} / \mathrm{kg}$ once daily. Our study revealed that the mean initial AZA dose in Crohn's disease and after modification was also lower than recommended. Neither was there any difference between the initial AZA dose and the dose after adjustment, based on 6-TGN concentrations, most likely for low AZA dose at baseline. The decision about the starting dose was made individually by a gastroenterologist. Various practical approaches among practitioners included thiopurine dosage, decisions about continuing thiopurines, and timing of metabolite assays. The other reason for lower AZA doses was the fact that, according to the previous studies, a lower dose of azathioprine is effective to induce and maintain remission in active Crohn's disease. Qian et al., in a prospective observational study, revealed that azathioprine, $1.5 \mathrm{mg} / \mathrm{kg} / \mathrm{d}$, combined with steroids was as effective as AZA $2.0 \mathrm{mg} / \mathrm{kg} / \mathrm{d}$ to induce remission of active $\mathrm{CD}$ in the first 6 months and to maintain remission of inactive $C D$ in the first 2 years, without higher recurrence rate of active CD [29]. Another Chinese study confirmed that observation [30].

The mean AZA dose in CU patients, both at the beginning and after modification, was also lower than recommended $(0.97,0.87$, and $2.0-2.5 \mathrm{mg} / \mathrm{kg} /$ day, respectively). The mean 6-TGN concentration was within the range (349, $47,11 \mathrm{pmol} / 8 \times 108$ erythrocytes). Hibi et al. revealed the same observation in adults [31]. Walker et al. [26] also showed that, in the paediatric IBD children, the AZA dose, sufficient to maintain remission, was $1.3 \pm 0.4 \mathrm{mg} / \mathrm{kg}$; after excluding children on biologics, the effective azathioprine dose was $1.4 \pm 0.5 \mathrm{mg} / \mathrm{kg}$. Apart from receiving low AZA doses, $75 \%$ of IBD patients in our study were in remission. Those results suggest that therapeutic thiopurine metabolites can be achieved with a dose lower than recommended, although, since TMPT activity was not determined at the beginning of treatment, we could not know then that lower AZA doses could be sufficient to maintain remission.

5.5. Side Effects. Myelotoxicity is one of the most serious thiopurine-induced side effects and may occur at any time during the treatment. It is strongly linked to low TPMT enzyme activity and high 6-TGN blood levels. Myelotoxicity may also occur with normal TPMT activity, necessitating regular full blood count monitoring in clinical practice. In a review of 66 studies, including more than 8,000 thiopurinetreated patients, the incidence rate of drug-induced myelotoxicity was $3 \%$ per patient year of treatment [32]. In our study, only one patient $(1.6 \%)$ developed leukopenia $(<3.5$ $\mathrm{WBCx} \times 10^{9}$ ), but it was not related to high 6-TG levels. None of the children developed azathioprine toxicity, as defined by abnormal liver function tests. That observation was similar to that in another study [26]. On the contrary, Pavlovska et al. reported a higher percentage of adverse effects [24]. The most common treatment-related complication was leucocytopaenia ( $42.9 \%$ ), followed by elevated transaminase levels (28.6\%), aphthous ulcers (14.3\%), and elevated amylase in serum (14.3\%).

We did not find any correlation between faecal calprotectin (FC) and 6T-GN levels. On the contrary, another study showed that, in patients with CD on AZA monotherapy, 6TGN concentrations within a defined range (250-450 $\mathrm{pmol} / 8 \times 10^{8}$ erythrocytes) were associated with significantly lower FC [33].

We acknowledge the limitations of this study, including the lack of TPMT activity tests, justified by cost and availability issues, the sample size, and the retrospective design.

Measurements of 6-TGN and 6-MMP levels in IBD and AIH patients on AZA/6-MP may help identify patients at risk for toxicity and provide an explanation for the ineffectiveness of the treatment, observed in some patients. Thiopurine metabolite measurements become more and more available, although their routine use is still limited by costs and laboratory gear.

In conclusion, timely measurements of thiopurine metabolites can be a useful tool for the identification of nonadherent patients before adding or switching to another drug. This method can also identify patients receiving too low or too high doses, enabling subsequent corrections of drug doses, as the patients with optimal 6-TGN levels presented a higher percentage of remission than those who were under- or overdosed.

\section{Data Availability}

Data are available on request through the authors themselves. Contact: bak-drabik@wp.pl

\section{Conflicts of Interest}

The authors declare that they have no conflicts of interest. 


\section{References}

[1] G. B. Elion, "The purine path to chemotherapy," Science, vol. 244, no. 4900, pp. 41-47, 1989.

[2] E. Sánchez Rodríguez, R. Ríos León, F. Mesonero Gismero, A. Albillos, and A. Lopez-Sanroman, "Clinical experience of optimising thiopurine use through metabolite measurement in inflammatory bowel disease," Gastroenterología y Hepatología, vol. 41, no. 10, pp. 629-635, 2018.

[3] M. M. Alsous, A. F. Hawwa, C. Imrie et al., "Adherence to azathioprine/6-mercaptopurine in children and adolescents with inflammatory bowel diseases: a multimethod study," Canadian Journal of Gastroenterology \& Hepatology, vol. 2020, article 9562192, pp. 1-10, 2020.

[4] Y. González-Lama and J. P. Gisbert, "Monitoring thiopurine metabolites in inflammatory bowel disease," Frontline Gastroenterol, vol. 7, no. 4, pp. 301-307, 2016.

[5] A. J. Yarur, B. Gondal, A. Hirsch, B. Christensen, R. D. Cohen, and D. T. Rubin, "Higher thioguanine nucleotide metabolite levels are associated with better long-term outcomes in patients with inflammatory bowel diseases," Journal of Clinical Gastroenterology, vol. 52, no. 6, pp. 537-544, 2018.

[6] J. P. Gisbert and F. Gomollón, "Thiopurine-induced myelotoxicity in patients with inflammatory bowel disease: a review," The American Journal of Gastroenterology, vol. 103, no. 7, pp. 1783-1800, 2008, Epub 2008 Jun 28.

[7] D. R. Wong, M. J. Coenen, L. J. Derijks et al., "Early prediction of thiopurine-induced hepatotoxicity in inflammatory bowel disease," Alimentary Pharmacology \& Therapeutics, vol. 45, no. 3, pp. 391-402, 2017, Epub 2016 Dec 12.

[8] F. M. Ruemmele, G. Veres, K. L. Kolho et al., "Consensus guidelines of ECCO/ESPGHAN on the medical management of pediatric Crohn's disease," Journal of Crohn's \& Colitis, vol. 8, no. 10, pp. 1179-1207, 2014, Epub 2014 Jun 6.

[9] D. Turner, F. M. Ruemmele, E. Orlanski-Meyer et al., "Management of paediatric ulcerative colitis, part 1: ambulatory care-an evidence-based guideline from European Crohn's and Colitis Organization and European Society of Paediatric Gastroenterology, Hepatology and Nutrition," Journal of Pediatric Gastroenterology and Nutrition, vol. 67, no. 2, pp. 257291, 2018.

[10] G. Mieli-Vergani, D. Vergani, U. Baumann et al., "Diagnosis and management of pediatric autoimmune liver disease: ESPGHAN hepatology committee position statement," Journal of Pediatric Gastroenterology and Nutrition, vol. 66, no. 2, pp. 345-360, 2018.

[11] J. Y. Chang and J. H. Cheon, "Thiopurine therapy in patients with inflammatory bowel disease: a focus on metabolism and pharmacogenetics," Digestive Diseases and Sciences, vol. 64, no. 9, pp. 2395-2403, 2019, Epub 2019 Jul 9.

[12] G. Stocco, M. Londero, A. Campanozzi et al., "Usefulness of the measurement of azathioprine metabolites in the assessment of non-adherence," Journal of Crohn's \& Colitis, vol. 4, no. 5, pp. 599-602, 2010.

[13] R. S. Bloomfeld and J. E. Onken, "Mercaptopurine metabolite results in clinical gastroenterology practice," Alimentary Pharmacology \& Therapeutics, vol. 17, no. 1, pp. 69-73, 2003.

[14] B. BOKEMEYER, A. TEML, C. ROGGEL et al., "Adherence to thiopurine treatment in out-patients with Crohn's disease," Alimentary Pharmacology \& Therapeutics, vol. 26, no. 2, pp. 217-225, 2007.
[15] S. Wright, D. S. Sanders, A. J. Lobo, and L. Lennard, "Clinical significance of azathioprine active metabolite concentrations in inflammatory bowel disease," Gut, vol. 53, no. 8, pp. 1123-1128, 2004.

[16] K. A. Hommel, C. M. Davis, and R. N. Baldassano, "Objective versus subjective assessment of oral medication adherence in pediatric inflammatory bowel disease," Inflammatory Bowel Diseases, vol. 15, no. 4, pp. 589-593, 2009.

[17] K. A. Hommel, R. N. Greenley, M. H. Maddux, W. N. Gray, and L. M. Mackner, "Self-management in pediatric inflammatory bowel disease: a clinical report of the North American Society for Pediatric Gastroenterology, Hepatology, and Nutrition," Journal of Pediatric Gastroenterology and Nutrition, vol. 57, no. 2, pp. 250-257, 2013.

[18] L. J. J. DERIJKS, L. P. L. GILISSEN, P. M. HOOYMANS, and D. W. HOMMES, "Review article: thiopurines in inflammatory bowel disease," Alimentary Pharmacology \& Therapeutics, vol. 24, no. 5, pp. 715-729, 2006.

[19] R. B. Gearry and M. L. Barclay, "Azathioprine and 6mercaptopurine pharmacogenetics and metabolite monitoring in inflammatory bowel disease," Journal of Gastroenterology and Hepatology, vol. 20, no. 8, pp. 1149-1157, 2005.

[20] M. C. Dubinsky, S. Lamothe, H. Yang et al., "Pharmacogenomics and metabolite measurement for 6-mercaptopurine therapy in inflammatory bowel disease," Gastroenterology, vol. 118, no. 4, pp. 705-713, 2000.

[21] M. A. Sheiko, S. S. Sundaram, K. E. Capocelli, Z. Pan, A. M. McCoy, and C. L. Mack, "Outcomes in pediatric autoimmune hepatitis and significance of azathioprine metabolites," Journal of Pediatric Gastroenterology and Nutrition, vol. 65, no. 1, pp. 80-85, 2017.

[22] T. M. Nguyen, M. Daubard, C. le Gall, M. Larger, A. Lachaux, and R. Boulieu, "Monitoring of azathioprine metabolites in pediatric patients with autoimmune hepatitis," Therapeutic Drug Monitoring, vol. 32, no. 4, pp. 433-437, 2010.

[23] M. N. Lee, B. Kang, S. Y. Choi et al., "Relationship between azathioprine dosage, 6-thioguanine nucleotide levels, and therapeutic response in pediatric patients with IBD treated with azathioprine," Inflammatory Bowel Diseases, vol. 21, no. 5, pp. 1054-1062, 2015.

[24] K. Pavlovska, M. Petrushevska, K. Gjorgjievska et al., "Importance of 6-thioguanine nucleotide metabolite monitoring in inflammatory bowel disease patients treated with azathioprine," Pril (Makedon Akad Nauk Umet Odd Med Nauki)., vol. 40, no. 1, pp. 73-79, 2019.

[25] M. C. Dubinsky, E. Reyes, J. Ofman, C. F. Chiou, S. Wade, and W. J. Sandborn, "A cost-effectiveness analysis of alternative disease management strategies in patients with Crohn's disease treated with azathioprine or 6-mercaptopurine," The American Journal of Gastroenterology, vol. 100, no. 10, pp. 22392247, 2005.

[26] R. Walker, J. Kammermeier, R. Vora, and M. Mutalib, “Azathioprine dosing and metabolite measurement in pediatric inflammatory bowel disease: does one size fit all?," Annals of Gastroenterology, vol. 32, no. 4, pp. 387-391, 2019.

[27] P. W. Lowry, C. L. Franklin, A. L. Weaver et al., "Leucopenia resulting from a drug interaction between azathioprine or 6mercaptopurine and mesalamine, sulphasalazine, or balsalazide," Gut, vol. 49, no. 5, pp. 656-664, 2001.

[28] T. Dassopoulos, M. C. Dubinsky, J. L. Bentsen et al., "Randomised clinical trial: individualised vs. weight-based dosing of 
azathioprine in Crohn's disease," Alimentary Pharmacology \& Therapeutics, vol. 39, no. 2, pp. 163-175, 2014.

[29] X. Qian, T. Wang, J. Shen, and Z. Ran, "Low dose of azathioprine is effective to induce and maintain remission in active Crohn disease: a prospective observational study," Medicine (Baltimore), vol. 97, no. 34, article e11814, 2018.

[30] J. Wu, Y. Gao, C. Yang, X. Yang, X. Li, and S. Xiao, "Low-dose azathioprine is effective in maintaining remission among Chinese patients with Crohn's disease," Journal of Translational Medicine, vol. 11, no. 1, p. 235, 2013.

[31] T. Hibi, M. Naganuma, T. Kitahora, F. Kinjyo, and T. Shimoyama, "Low-dose azathioprine is effective and safe for maintenance of remission in patients with ulcerative colitis," Journal of Gastroenterology, vol. 38, no. 8, pp. 740-746, 2003.

[32] R. P. Luber, S. Honap, G. Cunningham, and P. M. Irving, "Can we predict the toxicity and response to thiopurines in inflammatory bowel diseases?," Front Med (Lausanne), vol. 6, p. 279, 2019.

[33] J. Essmann, C. Keil, O. Unruh, A. Otte, M. P. Manns, and O. Bachmann, "Fecal calprotectin is significantly linked to azathioprine metabolite concentrations in Crohn's disease," European Journal of Gastroenterology \& Hepatology, vol. 31, no. 1, pp. 99-108, 2019. 